\title{
Efecto de bacterias emulsificantes en la atenuación de la fitotoxicidad de suelos contaminados con petróleo intemperizado
}

\author{
Gilberto Morales-Guzmán ${ }^{1 *}$, Alejandro Alarcón ${ }^{1}$, Ronald Ferrera-Cerrato ${ }^{1}$, María del Carmen \\ Rivera-Cruz ${ }^{2}$, Luis Gilberto Torres-Bustillos ${ }^{3}$ \& Ma. Remedios Mendoza-López ${ }^{4}$
}

1. Área de Microbiología, Postgrado en Edafología, Colegio de Postgraduados, Carretera México-Texcoco km 36.5, Montecillo 56230, Estado de México, México; gmoralesg06@gmail.com, aalarconcp@gmail.com, rferreracerrato@gmail.com

2. Colegio de Postgraduados Campus Tabasco. Periférico Carlos A. Molina s/n km. 3.5 CP. 86570. H. Cárdenas, Tabasco, México; mariari@colpos.mx

3. Unidad Profesional Interdisciplinaria de Biotecnología-Instituto Politécnico Nacional (UPIBI-IPN), Av. Acueducto s.n. Colonia Barrio la Laguna Ticomán, México 07340 CD., México; 1torresbustillos@gmail.com

4. Unidad de Servicios de Apoyo en Resolución Analítica (SARA). Universidad Veracruzana, Dr. Luis Castelazo Ayala s/n, Col. Industrial-Animas, CP 91190, Xalapa, Veracruz, México; remendoza@uv.mx

* Correspondencia

Recibido 15-X-2019. Corregido 31-I-2020. Aceptado 30-III-2020.

\begin{abstract}
Effect of emulsifying bacteria on phytotoxicity attenuation of soils contaminated with weathered petroleum hydrocarbons. Introduction: Plants and microorganisms have been used as bioindicators to evaluate the toxicity of hydrocarbons in soils. Objective: This study evaluates the toxicity of a chronically-contaminated Gleysol with several concentrations of weathered petroleum hydrocarbons (WPH), collected from La Venta, Tabasco (Mexico), on the growth of Clitoria ternatea and the phytoxicity attenuation due to inoculation of emulsifying bacteria. Methods: Soils with 50 and $150 \mathrm{~g} \mathrm{WPH} \mathrm{kg}^{-1}$, and control soil with $0.15 \mathrm{~g} \mathrm{WPH} \mathrm{kg}^{-1}$ (biogenic origin) were utilized, as well as the inoculation of six emulsifying bacteria and their combination (consortium). The WPH-phytotoxicity was evaluated by considering plant height, dry biomass production (root, shoot, and total), leaf area, specific leaf area, and the efficiency of photosystem II (EPSII), after 30 days. Results: WPH did not affect plant height, but soil with $50 \mathrm{~g} \mathrm{WPH} \mathrm{kg}^{-1}$ diminished root and total dry weight, and leaf area, when compared to both control soil and soil with $150 \mathrm{~g} \mathrm{WPH} \mathrm{kg}^{-1}$. The strain Sml (Stenotrophomonas maltophilia C10S1) significantly increased shoot and total dry weight, while the strain Ro (Raoultella ornithinolytica $\mathrm{C} 5 \mathrm{~S} 3$ ) produced higher specific leaf area relative to uninoculated plants. In control soil, the bacterial consortium stimulated plant height. The strains Sm (Serratia marcescens C11S1) and Sm2 (S. marcescens C7S3) improved plant height and specific leaf area when compared to uninoculated plants in soil with $50 \mathrm{~g} \mathrm{WPH} \mathrm{kg}^{-1}$. In soil with $150 \mathrm{~g} \mathrm{WPH} \mathrm{kg}^{-1}$, strains Spa (Stenotrophomonas pavanii C5S3F) and Cfr (Citrobacter freundii C4S3) enhanced root and shoot dry weight, respectively. The EPSII was unaffected by soil contamination. Emulsifying bacteria reduced the phytotoxic effects of WP, but depending on the content of WPH in soils. Conclusions: Soil with $50 \mathrm{~g} \mathrm{WPH} \mathrm{kg}^{-1}$ showed the greatest phytotoxic effects on plant growth.

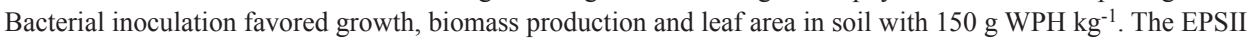
was not affected by WPH in soils.
\end{abstract}

Key words: Clitoria ternatea, PSII efficiency, Gleysol, bacteria, weathered petroleum.

Morales-Guzmán, G., Alarcón, A., Ferrera-Cerrato, R., Rivera-Cruz, M.C., Torres-Bustillos, L.G., \& Mendoza-López, M.R. (2020). Efecto de bacterias emulsificantes en la atenuación de la fitotoxicidad de suelos contaminados con petróleo intemperizado. Revista de Biología Tropical, 68(2), 692-703. 
A nivel mundial el petróleo es una de las fuentes de energía más importantes para la economía. En el estado de Tabasco (México), la explotación de este recurso no renovable ha originado impactos negativos en el ambiente, tan solo entre 2000 y 2017, se registraron 8581 derrames de petróleo en México, de los cuales el $9.7 \%$ ocurrieron en Tabasco (SEMARNAT, 2018). Las principales causas de estos derrames están asociadas a las actividades de extracción y transporte del petróleo (Trujillo-Narcía, Rivera-Cruz, Trujillo-Rivera, \& Garrigos, 2018). Una vez liberados en el medio ambiente, los hidrocarburos del petróleo pueden ser adsorbidos en la matriz del suelo, haciéndolos menos biodisponibles para microorganismos y plantas (Maletić, Dalmacija, Rončević, Agbaba, \& Perović, 2011; Ramadass, Megharaj, Venkateswarlu, \& Naidu, 2018).

Los hidrocarburos totales del petróleo intemperizados (HTPI) son hidrocarburos totales del petróleo (HTP) que fueron expuestos por largos periodos de tiempo a las condiciones ambientales (inundación-sequía, temperaturas extremas, lixiviación y radiación solar, etc.), los cuales son más complejos, recalcitrantes y menos biodisponibles (Maletić et al., 2011; Rivera-Cruz, Trujillo-Narcía, Miranda, \& Maldonado, 2016; Lustosa et al., 2018).

Los HTPI alteran las propiedades físicas y químicas del suelo y por consiguiente pueden causar toxicidad tanto a las plantas como a los microorganismos de la rizosfera (Adams, Kanga-Leyva, Guzmán-Osorio, \& EscalanteEspinosa, 2011; Pernía et al., 2018); por lo que la toxicidad puede ser un criterio importante para evaluar el impacto de los HTPI en los suelos y en los procesos de remediación (Alexander, 1999). La fitotoxicidad en plantas sensibles se manifiesta como necrosis del follaje y raíz, ocasionando la disminución en la actividad fotosintética, reducción de la biomasa aérea, e incluso la muerte de la planta (Chaineau, Morel, \& Oudot, 1997; Pernía et al., 2018). Las especies utilizadas en la fitorremediación incluyen gramíneas y leguminosas (Khan, Afzal, Iqbal, \& Khan, 2013; Khan, Biswas, Smith, Naidu, \& Megharaj, 2018); aunque las leguminosas son más susceptibles que las gramíneas a sufrir daños severos por exposición a los HTPI en el suelo, por lo que pueden ser utilizadas como indicadoras de toxicidad inducida por estos compuestos (Khan et al., 2013; Morales-Guzmán et al., 2017).

Los bioensayos de fitotoxicidad son utilizados para medir la respuesta fisiológica de las plantas a diferentes concentraciones de HTPI y para evaluar la eficacia de los procesos de remediación (Balseiro-Romero \& Monterroso, 2014; Cartmill, Cartmill, \& Alarcón, 2014; Pernía et al., 2018). La toxicidad de los hidrocarburos del petróleo hacia las plantas puede ser atenuada mediante el uso de rizobacterias y microorganismos productores de surfactantes, degradadoras de hidrocarburos y productoras de fitohormonas (Khan et al., 2013; Onur, Yilmaz, \& Icgen, 2015; Xie et al., 2018). Las bacterias productoras de surfactantes mejoran la dispersión y favorecen la remoción de los compuestos del petróleo, mediante el proceso de emulsificación permitiendo que sean utilizados como co-sustrato, y fuente de energía para satisfacer el metabolismo microbiano e incrementar la degradación de los hidrocarburos del petróleo, favoreciendo el crecimiento de las plantas (Morales-Guzmán et al., 2017; Reddy, Karegoudar, \& Nayak, 2018; Cecotti, Coppote1li, Mora, Viera, \& Morelli, 2018).

Clitoria ternatea L. (Fabaceae) es una leguminosa que establece asociación simbiótica con bacterias fijadoras de nitrógeno, además, promueve la fertilidad de los suelos y es tolerante a las concentraciones de los HP (Sangabriel et al., 2006; Jamil, Zairi, Nasim, \& Pa'ee, 2018; Somtrakoon et al., 2018). Debido a su importancia ambiental en la restauración de suelos degradados y contaminados, C. ternatea y bacterias emulsificantes e hidrocarbonoclastas (bacterias degradadoras de hidrocarburos del petróleo), constituyen una alternativa biológica de bajo costo y amigable con el ambiente en la atenuación de la toxicidad de los suelos crónicamente contaminados. El objetivo de la presente investigación fue evaluar la fitotoxicidad en el crecimiento de C. ternatea, y el efecto de la inoculación de bacterias emulsificantes en 
la atenuación de la toxicidad inducida por un Gleysol contaminado de origen, con diferentes concentraciones de petróleo intemperizado, recolectado en La Venta, Tabasco (México).

\section{MATERIALES Y MÉTODOS}

Área de estudio y recolección del suelo: Se seleccionó un suelo (Gleysol) contaminado con dos concentraciones de petróleo intemperizado: A) $50 \mathrm{~g} \mathrm{~kg}^{-1}$ y B) $150 \mathrm{~g} \mathrm{~kg}^{-1}$ (Rivera-Cruz et al. 2005; Trujillo et al., 2018), recolectados en el ejido José Narciso Rovirosa en el área aledaña al sur del Complejo Procesador de Gas La Venta, en el estado de Tabasco (1804'39.83" $\mathrm{N} \&$ \& 9402'39.42” W y $18^{\circ} 04^{\prime} 39.22^{\prime}$ ” N \& 9402'38.46" W). Además, se utilizó un suelo testigo con las mismas características pedogenéticas $\left(0.150 \mathrm{~g} \mathrm{~kg}^{-1}\right.$ de hidrocarburos de origen biogénico) recolectado de él ejido Blasillo Huimanguillo Tabasco (1803'9.35” N \& 93 $95^{\circ}$ '59.34" W). En cada uno de los tres suelos se recolectó $200 \mathrm{~kg}^{-1}$ del horizonte superficial $(0-30 \mathrm{~cm})$ mediante un muestreo puntual. A cada muestra, se le determinó y registró conforme NOM-021-RECNAT-2000 (Diario Oficial de la Federación [DOF], 2002) (Tabla 1), norma mexicana que establece las especificaciones de fertilidad, salinidad y clasificación de suelos.

Análisis de hidrocarburos totales del petróleo: El contenido de los HTPI de las muestras recolectadas se extrajo en equipo Soxhlet usando el solvente diclometano al 99.5\% de pureza (Sigma-Aldrich, MO, EUA), por el método EPA 3540B (United States Enviromental Protection Agency [USEPA], 1994). El pH de las muestras de suelo se ajustó a 2.0 con $\mathrm{HCl}$ concentrado y, posteriormente se secaron con $\mathrm{MgSO}_{4}$. El solvente se evaporó utilizando un rotavapor y el extracto se cuantificó por gravimetría $\left(\mathrm{g} \mathrm{kg}^{-1}\right)$ con una balanza analítica (Sartorius, modelo Analytic AC 210S, IL, EUA), según el método NMX-AA-134SCFI-2006 (DOF, 2006).

Material microbiológico: Se utilizaron seis cepas, que pertenecen a la colección del Área de Microbiología de Suelos, Colegio de Postgraduados, Montecillo, Estado de México. Las cuales fueron aisladas de suelos contaminados con HTPI: Serratia marcescens C11S1 (Sm), Citrobacter freundii C4S3 (Cfr), Serratia marcescens $\mathrm{C} 7 \mathrm{~S} 3$ (Sm2), Raoultella ornithinolytica C5S3 (Ro), Stenotrophomonas pavanii C5S3F (Spa), Stenotrophomonas maltophilia C10S1 (Sml), y un consorcio integrado por estas seis bacterias (CS), (Morales-Guzmán et al., 2017).

Preparación de los inóculos: Cada cepa fue cultivada en medio Luria-Bertani (g/L: 10 Triptona, 5 extracto de levadura, $5 \mathrm{NaCl}$, 1 L-triptofano), a $28^{\circ} \mathrm{C}$ por $72 \mathrm{~h}$. Los cultivos obtenidos se centrifugaron a $7000 \times \mathrm{g}$ durante

TABLA 1

Características fisícas y químicas de los tres suelos en estudio

TABLA 1

Physical and chemical characteristics of the three soils under study

\begin{tabular}{lccccccc}
$\begin{array}{c}\text { Suelo } \\
\text { HTPI g kg-1 }\end{array}$ & $\mathrm{pH}$ & $\begin{array}{c}\mathrm{MO} \\
\%\end{array}$ & $\begin{array}{c}\mathrm{CE} \\
\mathrm{dSm}^{-1}\end{array}$ & $\begin{array}{c}\mathrm{CIC} \\
\mathrm{cmol}(+) \mathrm{kg}^{-1}\end{array}$ & $\begin{array}{c}\mathrm{K} \\
\mathrm{mg} \mathrm{kg}^{-1}\end{array}$ & $\begin{array}{c}\text { N-total } \\
\%\end{array}$ & $\begin{array}{c}\text { Textura } \\
\%\end{array}$ \\
0.150 & 6.5 & 8.60 & 1.12 & 57.0 & 192 & 0.49 & Franco arcillosa \\
50 & 5.0 & 7.90 & 4.73 & 29.9 & 184 & 0.39 & Franco arcilloso arenosa \\
150 & 5.5 & 26.2 & 2.93 & 62.3 & 312 & 0.59 & Franco arcilloso arenosa \\
\hline
\end{tabular}

$\mathrm{MO}=$ materia orgánica, $\mathrm{CE}=$ conductividad eléctrica, $\mathrm{CIC}=$ capacidad de intercambio catiónico, $\mathrm{K}=$ potasio, $\mathrm{N}$-total $=$ nitrógeno total, HTPI = hidrocarburos totales del petróleo. Los resultados fueron comparados con los rangos de referencia establecidos en la NOM-SEMARNAT-2000 (DOF, 2002). 
15 minutos para separar el pellet de las células bacterianas, que se lavó dos veces más con agua destilada estéril con el propósito de eliminar los nutrientes residuales. Cada inóculo se ajustó a una densidad óptica de $0.5\left(\mathrm{~A}_{600}\right)$, equivalente a $10^{8}$ UFC $\mathrm{mL}^{-1}$ (Morales-Guzmán et al., 2017). El consorcio bacteriano se preparó mezclando proporciones iguales $(0.5 \mathrm{~mL})$ con el procedimiento anterior.

Establecimiento del experimento e inoculación: Las semillas de Clitoria ternatea fueron recolectadas en el estado de Veracruz, México. Para evaluar la germinación de las semillas, se tomó como criterio de su emergencia la aparición de la radícula (Sangabriel et al., 2006), las cuales mostraron $95 \%$ de germinación. La siembra se realizó bajo condiciones de invernadero (temperatura de $13-24{ }^{\circ} \mathrm{C}$ y humedad relativa $38-83 \%$ ), en semilleros de poliestireno con 10 cavidades por tratamiento, cada cavidad fue considerada como una unidad experimental (UE). En cada UE se sembraron dos semillas en un sustrato conformado por cada suelo (testigo, 50 y $150 \mathrm{~g} \mathrm{~kg}^{-1}$ ), las cuales fueron inoculadas con $5 \mathrm{~mL}$ del respectivo inóculo bacteriano. Cada UE se mantuvo a humedad constante (28-30\%).

Variables de crecimiento vegetal: Después de 30 días, las plantas fueron cosechadas para medir la altura (distancia en $\mathrm{cm}$ desde la superficie del suelo al ápice del tallo principal), el área foliar y la biomasa seca total. El área foliar $\left(\mathrm{cm}^{2}\right)$ se determinó con un medidor LI-3000 (LI-COR Biosciences, NE, EUA). El área foliar específica se estimó dividiendo el área foliar, entre el peso seco de las hojas $\left(\mathrm{cm}^{2}\right.$ $\left.\mathrm{g}^{-1}\right)$. Las hojas, tallos y raíces se secaron en un horno (FELISA, Modelo 242-A, México D.F., México) a $70{ }^{\circ} \mathrm{C}$ por $72 \mathrm{~h}$ y posteriormente se pesó en una balanza analítica (Sangabriel et al., 2006).

Eficiencia fotoquímica del fotosistema II: A los 30 días, se evaluó la eficiencia fotoquímica del fotosistema II (Fv/Fm) se evaluó con un fluorómetro portátil (fluorómetro
OS30p+, Opti-Sciences, NH, EUA) a temperatura ambiente. Para ello, se seleccionó la hoja recientemente madura de cada planta y se cubrió previamente con un clip foliar por 15 min para adaptarla a la oscuridad (Bramley, Wasley, King, Powell, \& Robinson, 2014). La emisión de fluorescencia de la clorofila del fotosistema II (PSII) es el rendimiento cuántico máximo para la fotoquímica primaria cuando todos los centros de reacción del PSII están oxidados o "abiertos", expresado como la relación de fluorescencia variable y fluorescencia máxima (Fv/Fm) (Bramley et al., 2014).

Tratamientos y análisis estadístico: $\mathrm{El}$ experimento tuvo un diseño factorial $3 \times 8 \times$ 1 , con tres niveles de contaminación del suelo (testigo, 50 y $150 \mathrm{~g} \mathrm{~kg}^{-1}$ ), y ocho niveles de inoculación (Sm, Cfr, Sm2, Ro, Spa, Sml, y CS, más un tratamiento testigo), obteniéndose un total de 24 tratamientos con 6 repeticiones cada uno. Se realizó un análisis de varianza y a una prueba de comparación de medias del tipo LSD $(\alpha=0.05)$, utilizando el programa estadístico SAS (Statistical Analysis System, 2007), para todas las variables.

\section{RESULTADOS}

Crecimiento y producción de biomasa vegetal de Clitoria ternatea: En la presente investigación el factor concentración de los HTPI y el factor inoculación bacteriana no produjeron diferencias significativas en la variable altura de C. ternatea (Fig. 1A, Fig. 1B). El factor interacción, mostró diferencias estadísticas significativas $(\mathrm{P}<0.05)$ donde el suelo con $50 \mathrm{~g}$ HTPI kg-1 inoculado con la cepa $\mathrm{Sm} 2$ produjo mayor altura de planta con respecto al testigo sin inocular. Así mismo, el consorcio bacteriano estimuló la altura de las plantas crecidas en el suelo con $0.150 \mathrm{~g} \mathrm{~kg}^{-1}$ HTPI (testigo) (Fig. 1C).

La biomasa seca radical, aérea y total presentaron diferencias significativas $(\mathrm{P}<0.05)$ por efecto del factor concentración; las plantas crecidas en el suelo con $150 \mathrm{~g} \mathrm{~kg}^{-1}$ HTPI mostraron mayor biomasa seca radical, aérea y total con respecto al suelo testigo, por el 

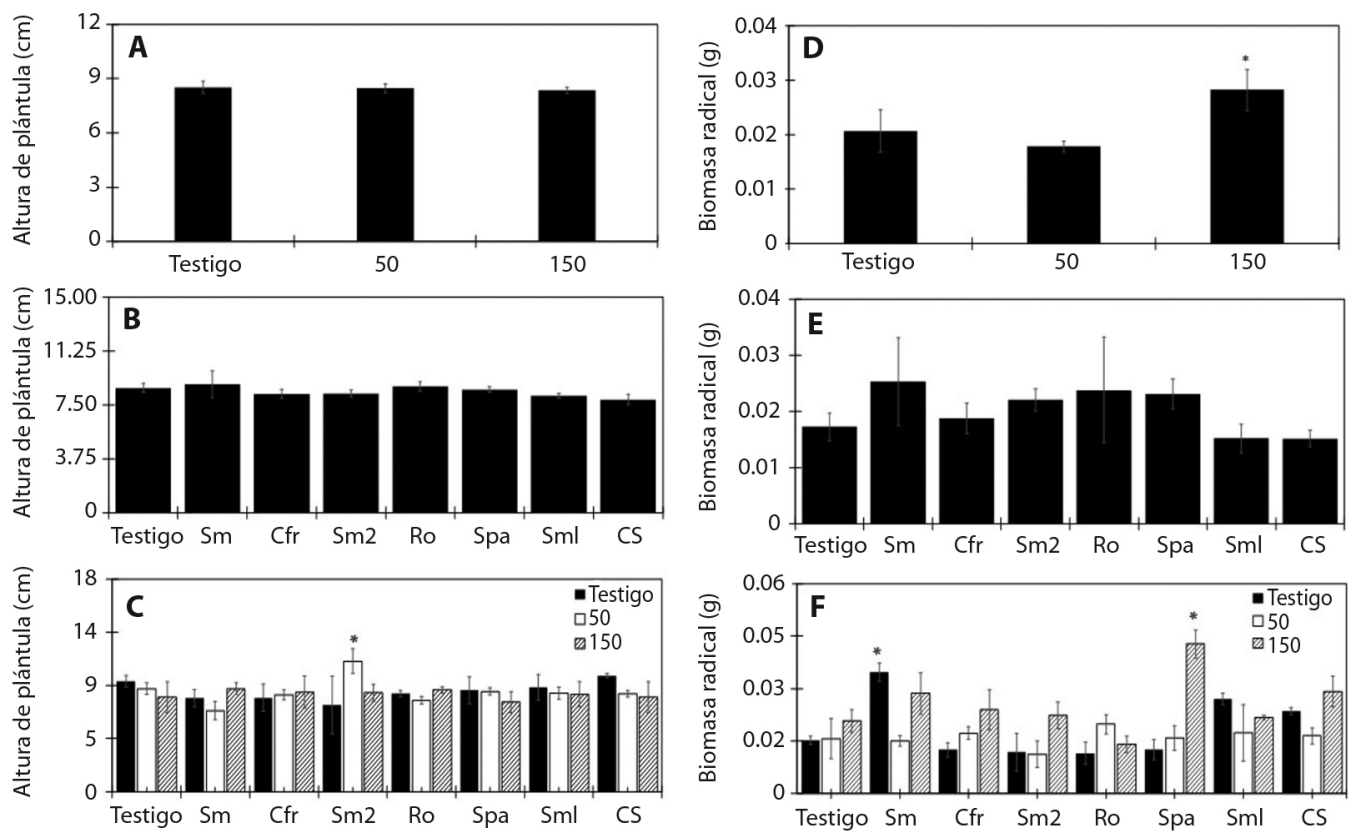

Fig. 1. Altura y biomasa seca radical de Clitoria ternatea después de 30 días de crecimiento en suelos con: (A) y (D), tres concentraciones de hidrocarburos de petróleo intemperizado (HPI) $(\mathrm{N}=24)$; (B) y (E), inoculación de bacterias emulsificantes $(\mathrm{N}=9)$; $(\mathbf{C})$ y $(\mathbf{F})$, la interacción [concentración de HPI] $\times$ [inoculación bacteriana] $(\mathrm{N}=3)$. Medias \pm error estándar; barras con diferentes asteriscos son estadísticamente diferentes (LSD, $\alpha=0.05$ ). Abreviaturas: Serratia marcescens C11S1 (Sm), Citrobacter freundii C4S3 (Cfr), Serratia marcescens C7S3 (Sm2), Raoultella ornithinolytica C5S3 (Ro), Stenotrophomonas pavanii C5S3F (Spa), Stenotrophomonas maltophilia C10S1 (Sml), CS = consorcio bacteriano.

Fig. 1. Plant height and root dry biomass of Clitoria ternatea after 30 days of growth in soils: (A) and (D), with three concentrations of weathered petroleum hydrocarbons $(\mathrm{WPH})(\mathrm{N}=24) ;(\mathbf{B})$ and $(\mathbf{E})$, inoculated with emulsifying bacteria $(\mathrm{N}=9)$; $(\mathbf{C})$ and $(\mathbf{F})$, with the [WPH concentration] $\times$ [bacterial inoculation] interaction $(\mathrm{N}=3)$. Means \pm standard error; bars with different asterisks are statistically different (LSD, $\alpha=0.05)$. Abbreviations: Serratia marcescens C11S1 (Sm), Citrobacter freundii C4S3 (Cfr), Serratia marcescens C7S3 (Sm2), Raoultella ornithinolytica C5S3 (Ro), Stenotrophomonas pavanii C5S3F (Spa), Stenotrophomonas maltophilia C10S1 (Sml), CS = bacterial consortium.

contario el suelo con $50 \mathrm{~g} \mathrm{~kg}^{-1}$ HTPI redujo en promedio $15 \%$ la biomasa vegetal (Fig. 1D, Fig. 2A, 2D). En contraste, la inoculación bacteriana solo mostró diferencias significativas $(\mathrm{P}<0.05)$ en la biomasa seca aérea y total (Fig. 2B, Fig. 2E), destacando la cepa Sml al incrementar $17 \%$ la biomasa vegetal respecto a las plantas sin inoculación. En cuanto al efecto por interacción de los dos factores (inoculación bacteriana y concentración hidrocarburo), se observaron diferencias significativas $(\mathrm{P}<0.05)$ en la biomasa seca radical, aérea y total (Fig. 1F, Fig. 2C, Fig. 2F). El suelo con $150 \mathrm{~g} \mathrm{~kg}^{-1}$ HTPI inoculado con las cepas Spa, Sm, Cs y
Cfr promovieron la mayor biomasa seca radical con respecto al testigo. así mismo, el suelo con $50 \mathrm{~g} \mathrm{~kg}^{-1}$ HTPI inoculado con la cepa Ro, Spa y $\mathrm{Sml}$ mostró la mayor biomasa seca radical con respecto al testigo no inoculado. La inoculación del suelo testigo con las cepas $\mathrm{Sm}, \mathrm{Sm} 2$ y $\mathrm{Sml}$ favorecieron la producción de biomasa seca radical y aérea con respecto al suelo testigo no inoculado.

El área foliar fue estadísticamente mayor $(\mathrm{P}<0.05)$ en la dosis más alta $\left(150 \mathrm{~g} \mathrm{~kg}^{-1}\right.$ HTPI) respeto al testigo (Fig. 3A). Para el caso del factor interacción la mayor diferencia se encontró en el tratamiento testigo inoculado 

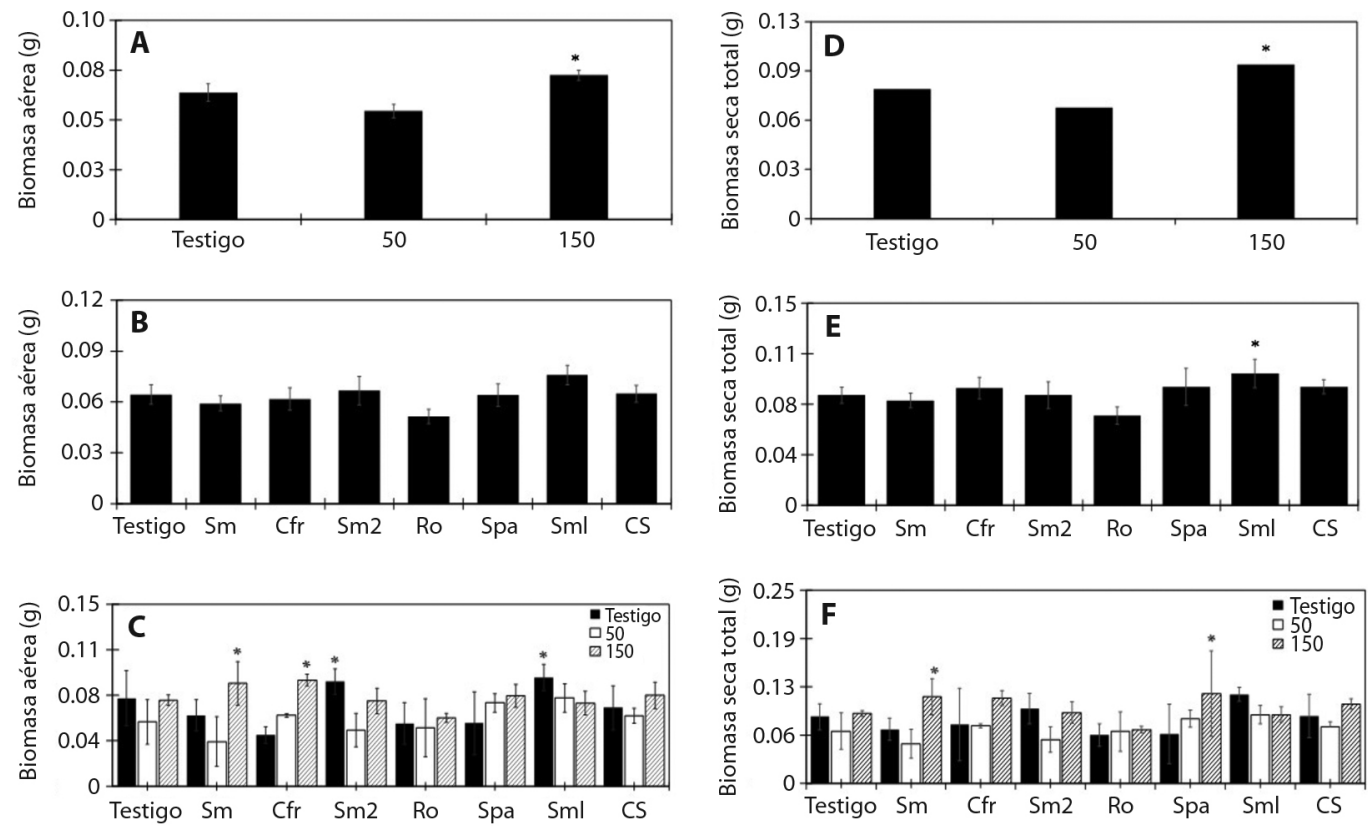

Fig. 2. Biomasa seca aérea y total de Clitoria ternatea después de 30 días de crecimiento en suelos con: (A) y (D), tres concentraciones de hidrocarburos de petróleo intemperizado (HPI) $(\mathrm{N}=24)$; (B) y $(\mathbf{E})$, inoculación de bacterias emulsificantes $(\mathrm{N}=9)$; $(\mathbf{C})$ y $(\mathbf{F})$, la interacción [concentración de HPI] $\times$ [inoculación bacteriana] $(\mathrm{N}=3)$. Medias \pm error estándar; barras con diferentes asteriscos son estadísticamente diferentes (LSD, $\alpha=0.05$ ). Abreviaturas: Serratia marcescens C11S1 (Sm), Citrobacter freundii C4S3 (Cfr), Serratia marcescens C7S3 (Sm2), Raoultella ornithinolytica C5S3 (Ro), Stenotrophomonas pavanii C5S3F (Spa), Stenotrophomonas maltophilia C10S1 (Sml), CS = consorcio bacteriano.

Fig. 2. Shoot (aerial) and total dry biomass of Clitoria ternatea after 30 days of growth in soils: (A) and (D), with three concentrations of weathered petroleum hydrocarbons $(\mathrm{WPH})(\mathrm{N}=24)$; $(\mathbf{B})$ and $(\mathbf{E})$, inoculated with emulsifying bacteria $(\mathrm{N}=9)$; $(\mathbf{C})$ and $(\mathbf{F})$, with the [WPH concentration] $\times$ [bacterial inoculation] interaction $(\mathrm{N}=3)$. Means \pm standard error; bars with different asterisks are statistically different (LSD, $\alpha=0.05)$. Abbreviations: Serratia marcescens C11S1 (Sm), Citrobacter freundii C4S3 (Cfr), Serratia marcescens C7S3 (Sm2), Raoultella ornithinolytica C5S3 (Ro), Stenotrophomonas pavanii C5S3F (Spa), Stenotrophomonas maltophilia $\mathrm{C} 10 \mathrm{~S} 1$ (Sml), CS = bacterial consortium.

respecto al resto de los tratamientos (Fig. 3C), en comparación al factor inoculación que no mostró diferencias significativas (Fig. 3B).

El área foliar de las plantas fue estadísticamente mayor $(\mathrm{P}<0.05)$ en el suelo con 150 $\mathrm{g} \mathrm{kg}^{-1}$ HTPI respecto al suelo testigo; por el contrario, la concentración de $50 \mathrm{~g} \mathrm{~kg}^{-1}$ HTPI redujo en $13 \%$ el área foliar (Fig. 3A). En cuanto a la inoculación, las cepas bacterianas Sm, y Ro mostraron un incremento del $10 \%$, del área foliar con respecto al testigo sin inoculación, aunque no se observó diferencias significativas entre los tratamientos (Fig. 3B). En cuanto a la interacción, del suelo testigo inoculado con las cepas $\mathrm{Sm} 2$, y $\mathrm{Sml}$ incrementaron significativamente el área foliar $(\mathrm{P}<0.05)$ con respecto al suelo testigo sin inoculación y al resto de los tratamientos. En el caso del suelo con $150 \mathrm{~g} \mathrm{~kg}^{-1}$ HTPI inoculado con las cepas $\mathrm{Sm} 2$, y Ro, incrementaron el área foliar. En lo que respecta al área foliar específica, el factor concentración no mostró diferencias significativas entre los tratamientos (Fig. 3D). En contraste, la interacción de los factores mostró diferencias estadísticas significativas ( $\mathrm{P}$ $<0.05$ ) (Fig. 3F), destacando el suelo con $50 \mathrm{~g}$ $\mathrm{kg}^{-1}$ HTPI inoculado con las cepas Sm y Sm2 al presentar la mayor área foliar (99 y $767 \%$ ) con respecto al suelo testigo inoculado y no inoculado (Fig. 3F). 

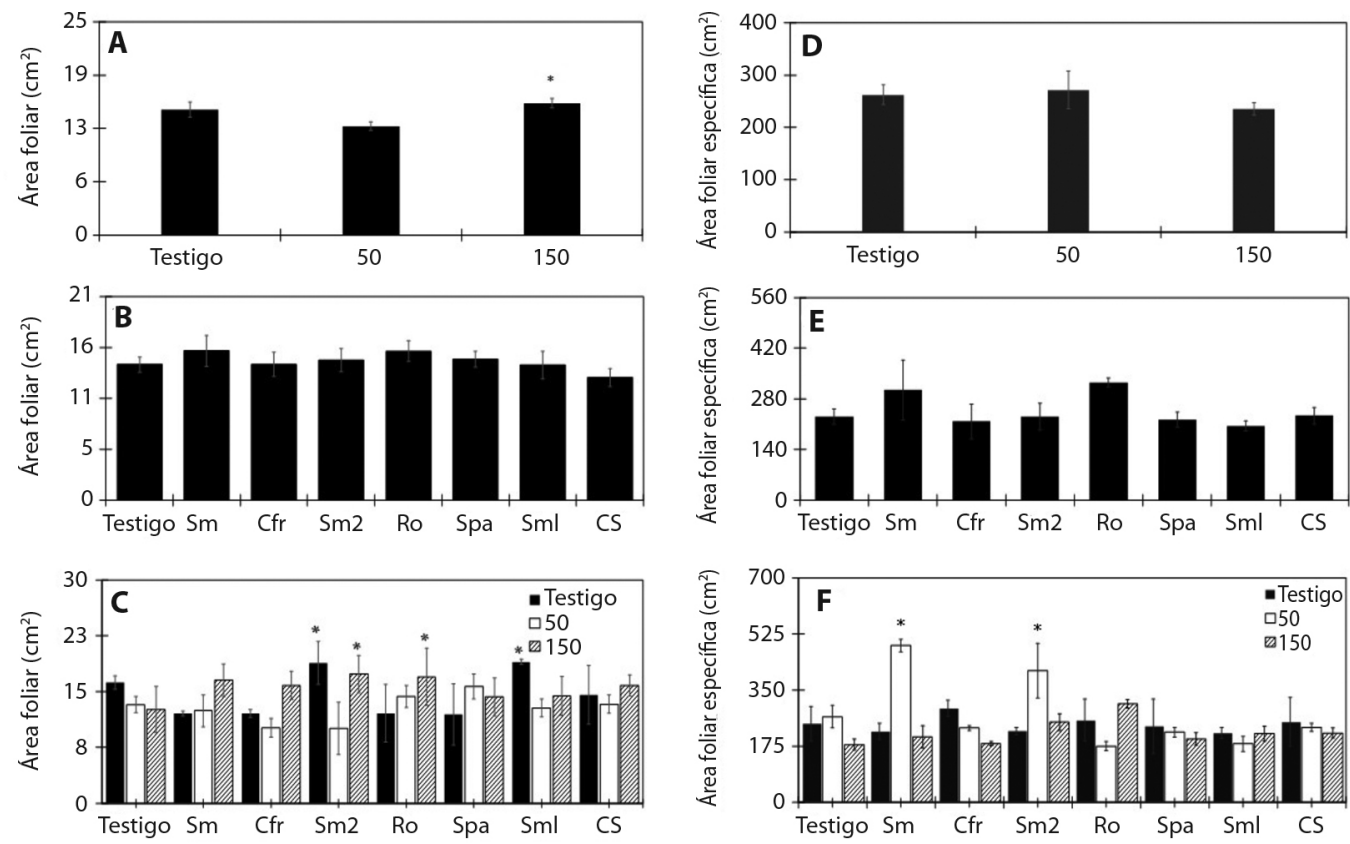

Fig. 3. Área foliar y área foliar específica de Clitoria ternatea después de 30 días de crecimiento en suelos con: (A) y (D), tres concentraciones de hidrocarburos de petróleo intemperizado (HPI) $(\mathrm{N}=24)$; (B) y (E), inoculación de bacterias emulsificantes $(\mathrm{N}=9)$; $(\mathbf{C})$ y $(\mathbf{F})$, la interacción [concentración de HPI] $\times$ [inoculación bacteriana] $(\mathrm{N}=3)$. Medias \pm error estándar; barras con diferentes asteriscos son estadísticamente diferentes (LSD, $\alpha=0.05)$. Abreviaturas: Serratia marcescens C11S1 (Sm), Citrobacter freundii C4S3 (Cfr), Serratia marcescens C7S3 (Sm2), Raoultella ornithinolytica C5S3 (Ro), Stenotrophomonas pavanii C5S3F (Spa), Stenotrophomonas maltophilia C10S1 (Sml), CS = consorcio bacteriano.

Fig. 3. Leaf area and specific leaf area of Clitoria ternatea after 30 days of growth in soils: (A) and (D), with three concentrations of weathered petroleum hydrocarbons (WPH) $(\mathrm{N}=24)$; $(\mathbf{B})$ and $(\mathbf{E})$, inoculated with emulsifying bacteria $(\mathrm{N}=9)$; $(\mathbf{C})$ and $(\mathbf{F})$, with the [WPH concentration] $\times$ [bacterial inoculation] interaction $(\mathrm{N}=3)$. Means \pm standard error; bars with different asterisks are statistically different (LSD, $\alpha=0.05)$. Abbreviations: Serratia marcescens C11S1 (Sm), Citrobacter freundii C4S3 (Cfr), Serratia marcescens C7S3 (Sm2), Raoultella ornithinolytica C5S3 (Ro), Stenotrophomonas pavanii C5S3F (Spa), Stenotrophomonas maltophilia $\mathrm{C} 10 \mathrm{~S} 1$ (Sml), CS = bacterial consortium.

\section{Medición eficiencia fotosintética del}

PSII: El análisis de varianza para la eficiencia fotosintética del PSII (Fv/Fm) a los 32 días no mostró diferencias significativas por efecto de los factores (concentración HTPI, inoculación, e interacción de los dos factores; $\mathrm{P}>0.05$ en todos los casos) (Datos no presentados). Además, los valores oscilaron en un rango de 0.74 a 0.82 ; en el cual se consideran dentro de un rango óptimo de la eficiencia fotosintética del PSII, donde los centros de reacción del PSII no son inhibidos en la fotosíntesis.

\section{DISCUSIÓN}

Altura biomasa aérea, biomasa radical y biomasa total de plántulas de Clitoria ternatea: Las propiedades físicas y químicas de los suelos influyen en gran medida en el crecimiento de las plantas y el desarrollo de las raíces (Tabla 1), las cuales pueden estimular la actividad microbiana y favorecer la degradación de los hidrocarburos (Ma, Rajkumar, Rocha, Oliveira, \& Freitas, 2015). El suelo con el tratamiento de mayor dosis $\left(150 \mathrm{~g} \mathrm{~kg}^{-1}\right)$ 
estimuló el crecimiento y la producción de biomasa vegetal de $C$. ternatea. esto posiblemente se debió a su alto contenido de materia orgánica (26\%), la cual puede adsorber los hidrocarburos del petróleo y funcionar como base para la formación de ácidos húmicos y fúlvicos, los cueles incrementan la capacidad de intercambio catiónico y la concentración de iones $\mathrm{H}$, reduciendo así su toxicidad y aumentando los procesos de transformación u oxidación biológica (mineralización), en combinación con la planta (rizorremediación) (Tan \& Napamonbodi, 1979; Totsche, Danzer, \& Kogel-Knabner, 1997; Evans, 2000; Volke Sepúlveda \& Velasco-Trejo, 2002; Adams, Álvarez-Ovando, \& Castañón, 2015). En este sentido, Rodríguez-Rodríguez, Rivera-Cruz, Trujillo- Narcía, Almaráz-Suárez, \& Salgado-García (2016) atribuyen mayor biomasa aérea del pasto Leersia hexandra al proceso de hormesis producido por petróleo intemperizado, el cual lo inhibe en dosis mayores a $5.5 \mathrm{~g} \mathrm{~kg}^{-1}$. Efecto similar fue reportado por Adams et al. (2015), quienes observaron mayor biomasa en pasto Brachiaria humidicola expuestas a concentraciones menores de $4 \mathrm{~g} \mathrm{~kg}^{-1}$ de hidrocarburo intemperizados. La nula toxicidad observada en la mayor concentración de los HTPI (150 $\left.\mathrm{g} \mathrm{kg}^{-1}\right)$ en el crecimiento de $C$. ternatea también ha sido reportada en especies como: Poa foliosa, Leucaena leucocephala, Festuca arundinacea y Sorghum bicolor, expuestas a los hidrocarburos alifáticos y aromáticos $\left(\geq 5 \mathrm{~g} \mathrm{~kg}^{-1}\right)$ (Muratova et al., 2009; López-Ortíz et al., 2012; Bramley, Wasley, King, Powell, \& Robinson, 2014).

Por otra parte, la inoculación de las bacterias $\mathrm{Sm}, \mathrm{Sm} 2$, Ro, y Spa estimularon la producción de biomasa seca radical. Este resultado posiblemente se debió a que las bacterias tienen la capacidad de producir surfactantes que pueden aumentar la degradación de los HTPI y por ende reducir su toxicidad (Morales-Guzmán et al., 2017). Estudios previos han demostrado que Serratia marcescens y Stenotrophomonas maltophilia se caracterizan por tener actividad nitrogenasa, solubilizar fosfatos, y producir ácido indolacético (AIA); además de tener actividad ACC-desaminasa (Ahemad \&
Kibret, 2014). El AIA a menudo puede inducir un efecto benéfico sobre el crecimiento y el desarrollo de plantas.

La producción de la enzima ACC-desaminasa puede mejorar el estrés causado por los hidrocarburos, ya que reduce los niveles de etileno de la planta e induce un mayor crecimiento vegetal en especial de la biomasa radical que estimula el desarrollo de las poblaciones microbianas u otros mecanismos, tales como el aumento de la disponibilidad de nutrimentos esenciales como el nitrógeno, fósforo y potasio (Afzal, Yousaf, Reichenauer, Kuffner, \& Sessitsch, 2011; Balseiro-Romero \& Monterroso, 2014; Balseiro-Romero, Gkorezis, Kidd, Vangronsveld, \& Monterroso, 2016). En este sentido, Khan et al. (2013) mencionan que las bacterias con potencial de producir surfactantes pueden degradar hidrocarburos y con ello disminuir la fitotoxicidad que producen los mismos. Algunos estudios han demostrado que la inoculación de cepas bacterianas productoras de surfactantes y degradadoras de hidrocarburos como: Pseudomonas sp. ITRI53, Planomicrobium chinense, Burkholderia phytofirmans PsJN y Sphingobacterium sp., reducen la fitotoxicidad de los hidrocarburos al mejorar el crecimiento y desarrollo de raigrás, Casuarina equisetifolia, Vigna radiata y Withania somnifera al conferirles tolerancia a condiciones de estrés mediante la degradación de los contaminante (Díaz-Martínez, Alarcón, Ferrera-Cerrato, Suarez, \& García-Barradas, 2013; Das \& Tiwary, 2014; Kumar, Das, \& Asha, 2014). El efecto negativo de los HTPI en el área foliar y área foliar especifica se atribuye a la toxicidad inherente de los hidrocarburos no biodegradados y recalcitrantes (compuestos cíclicos, aromáticos, asfáltenos y resinas), los cuales causan disminución en el crecimiento de la raíz y, por ende, disminuyen la biomasa aérea. Además, que el hidrocarburo forme una capa fina sobre la raíz de las plantas impidiendo la absorción de los nutrientes y del agua, esenciales para crecimiento (Cartmill et al., 2014). Resultados similares han sido descritos para las plantas: Avena sativa, Hordeum vulgare, Chloris gayana, Triticum aestivum, Phragmites australis, 
Arabidopsis, Lolium multiflorum, Poa foliosa, Cucumis melo, Capsicum frutescens, Solanum lycopersicum, y Amaranthus hybridus cuando fueron expuestas a hidrocarburos aromáticos y poliaromáticos (Anoliefo \& Nwoko, 1995, Zhang, \& Xing, 2008; García, Ferrera, Almaráz, Rodríguez, \& García, 2013; Cartmill et al., 2014; Bramley-Alves et al., 2014, Zhu et al., 2015; Rodríguez-Rodríguez et al., 2016).

\section{Eficiencia fotosintética del PSII (Fv/} Fm): La eficiencia fotosintética del PSII (Fv/ Fm) ha sido un indicador de la máxima eficiencia cuántica de las reacciones fotoquímicas del PSII (Butler, 1978). En plantas sanas y no estresadas, los valores oscilan entre 0.74 a 0.84 (Genty et al., 1989). En el presente estudio, el rendimiento fotosintético de C. ternatea no se vio afectado, según lo observado, por la disminución de estos parámetros después de trasplantar las plántulas al suelo contaminado. Esto se debe a que los hidrocarburos han estado expuestos por más de 20 años a las condiciones ambientales (Rivera-Cruz \& Trujillo-Narcia et al., 2004; Trujillo-Narcía et al., 2018), permitiendo que las fracciones volátiles ya no estén presentes, prevaleciendo los hidrocarburos de fracción pesada. Estos resultados coinciden con los obtenidos por Shiri et al. (2015) y BramleyAlves et al. (2014) al no observar efectos negativos en Thellungiella salsuginea, y Poa foliosa expuesta a fenantreno $(25 \mathrm{mM})$ e hidrocarburos SAB sobre la relación Fv/Fm. Contrario a los resultados obtenidos en la presente investigación, Cevher-Keskin et al., 2018, reportaron una disminución en la relación Fv/Fm, respecto al aumento en las concentraciones de petróleo crudo (2.5 y $5 \%$ v/w) en Zea mays (42 días). En este sentido Jin et al., (2017) observaron una disminución significativa en la relación Fv/Fm, en las hojas de Cucurbita pepo tratadas con fenantreno expuestas a $0.005 \mathrm{~g} \mathrm{~L}^{-1}$ de fenantreno.

En la presente investigación, posiblemente las concentraciones de los HTPI no limitaron la absorción los nutrimentos ni del agua, aunado a la presencia de bacterias productoras surfactantes, degradadoras de hidrocarburos, productoras de fitohormonas y productoras de la enzima ACC-desaminasa reducen los niveles de etileno de la planta, lo cual confiere resistencia al estrés causado por los HTPI y contribuyendo así, a la atenuación de la toxicidad de los mismos y a la supervivencia de las plantas ante esas condiciones. El uso de bacterias individuales o consorcios con capacidades emulsificantes y degradadoras de hidrocarburos que favorezcan la adaptación y la promoción del crecimiento de las plantas, y que coadyuven a la atenuación de la toxicidad de los hidrocarburos intemperizados, constituye una alternativa biológica de bajo costo para sitios crónicamente contaminados con hidrocarburos del petróleo.

Declaración de ética: los autores declaran que todos están de acuerdo con esta publicación y que han hecho aportes que justifican su autoría; que no hay conflicto de interés de ningún tipo; y que han cumplido con todos los requisitos y procedimientos éticos y legales pertinentes. Todas las fuentes de financiamiento se detallan plena y claramente en la sección de agradecimientos. El respectivo documento legal firmado se encuentra en los archivos de la revista.

\section{AGRADECIMIENTOS}

Esta investigación fue financiada por la beca SEP-CONACYT 79456. G. Morales-Guzmán agradece al CONACYT (Consejo Mexicano de la Ciencia y Tecnología) por el apoyo financiero durante su programa de doctorado.

\section{RESUMEN}

Introducción: Las plantas y los microorganismos se han utilizado como bioindicadores de la toxicidad inducida por hidrocarburos presentes en los suelos. Objetivo: El presente trabajo evaluó la toxicidad de un Gleysol contaminado de origen con diferentes concentraciones de petróleo intemperizado, recolectado en la Venta Tabasco (México), en el crecimiento de Clitoria ternatea, y la atenuación de la fitotoxicidad con la inoculación de bacterias emulsificantes. Metodología: Se usaron suelos con 50 y 150 g HTPI kg-1, y un suelo testigo con $0.15 \mathrm{~g} \mathrm{HTPI} \mathrm{kg}^{-1}$ (origen biogénico), y la inoculación de seis bacterias 
emulsificantes y su combinación (consorcio). La fitotoxicidad de los HTPI se evaluó considerando la altura, la biomasa seca (radical, aérea y total), el área foliar, el área foliar específica, y la eficiencia del fotosistema II (EPSII), a los 30 días. Resultados: Los HTPI no afectaron la altura, pero el suelo con $50 \mathrm{~g} \mathrm{HTPI} \mathrm{kg}^{-1}$ redujo la biomasa seca radical y total, y el área foliar con respecto a las plantas en los suelos testigo y con $150 \mathrm{~g} \mathrm{HTPI} \mathrm{kg}^{-1}$. La cepa Sml (Stenotrophomonas maltophilia C10S1) incrementó significativamente la biomasa seca total; la cepa Ro (Raoultella ornithinolytica $\mathrm{C} 5 \mathrm{~S} 3$ ) produjo mayor área foliar específica con respecto a plantas no inoculadas. En el suelo testigo, el consorcio bacteriano estimuló la altura; las cepas Sm (Serratia marcescens C11S1) y Sm2 (S. marcescens C7S3) mejoraron la altura y el área foliar específica con respecto a plantas no inoculadas, en el suelo con $50 \mathrm{~g}$ HTPI kg-1. En el suelo con $150 \mathrm{~g}$ HTPI $\mathrm{kg}^{-1}$, las cepas Spa (Stenotrophomonas pavanii $\mathrm{C} 5 \mathrm{~S} 3 \mathrm{~F}$ ) y Cfr (Citrobacter freundii C4S3) incrementaron la biomasa seca radical y aérea, respectivamente. La EPSII no fue afectada por la contaminación de los suelos. Las bacterias emulsificantes redujeron la fitotoxicidad de HTPI, pero dependiendo de su contenido en los suelos. Conclusiones: El suelo con $50 \mathrm{~g} \mathrm{HTPI} \mathrm{kg}^{-1}$ mostró mayor toxicidad en el crecimiento de las plantas. La inoculación bacteriana favoreció el crecimiento, producción de biomasa, y área foliar en el suelo con $150 \mathrm{~g}$ HTPI $\mathrm{kg}^{-1}$. La EPSII no fue afectada por la presencia de HTPI en el suelo.

Palabras clave: Clitoria ternatea, eficiencia fotosintética del PSII, Gleysol, bacterias.

\section{REFERENCIAS}

Adams, R.H., Álvarez-Ovando, A.L., \& Castañón, N. (2015). Efecto de la concentración de hidrocarburos sobre la producción del pasto (Brachiaria humidicola) en Texistepec, Veracruz. Phyton (Buenos Aires), 84(1), 222-232.

Adams, R.H., Kanga-Leyva, K., Guzmán-Osorio, F.J., \& Escalante-Espinosa, E. (2011). Comparison of moisture management methods for the bioremediation of hydrocarbon contaminated soil. African Journal of Biotechnology, 10(3), 394-404.

Afzal, M., Yousaf, S., Reichenauer, T.G., Kuffner, M., \& Sessitsch, A. (2011). Soil type affects plant colonization, activity and catabolic gene expression of inoculated bacterial strains during phytoremediation of diesel. Journal of Hazardous Materials, 186(2-3), 1568-1575.

Ahemad, M., \& Kibret, M. (2014). Mechanisms and applications of plant growth promoting rhizobacteria: current perspective. Journal of King Saud UniversityScience, 26(1), 1-20.
Alexander, M. (1999). Biodegradation and bioremediation (2nd ed.). San Diego, CA: Academic Press.

Anoliefo, G.O., \& Vwioko, D.E. (1995). Effects of spent lubricating oil on the growth of Capsicum annum L. and Lycopersicon esculentum Miller. Environmental Pollution, 88(3), 361-364.

Balseiro-Romero, M., \& Monterroso, C. (2014). Phytotoxicity of fuel to crop plants: influence of soil properties, fuel type, and plant tolerance. Toxicological \& Environmental Chemistry, 96(8), 1162-1173.

Balseiro-Romero, M., Gkorezis, P., Kidd, P.S., Vangronsveld, J., \& Monterroso, C. (2016). Enhanced degradation of diesel in the rhizosphere of Lupinus luteus after inoculation with diesel-degrading and plant growth-promoting bacterial strains. Journal of Environmental Quality, 45(3), 924-932.

Bramley, A.J., Wasley, J., King, C.K., Powell, S., \& Robinson, S.A. (2014). Phytoremediation of hydrocarbon contaminants in subantarctic soils: An effective management option. Journal of Environmental Management, 142, 60-69.

Butler, W.L. (1978). Energy distribution in the photochemical apparatus of photosynthesis. Annual Review of Plant Physiology, 29(1), 345-378.

Cartmill, A.D., Cartmill, D.L., \& Alarcón, A. (2014). Controlled release fertilizer increased phytoremediation of petroleum-contaminated sandy soil. International Journal of Phytoremediation, 16(3), 285-301.

Cecotti, M., Coppotelli, B.M., Mora, V.C., Viera, M., \& Morelli, I.S. (2018). Efficiency of surfactantenhanced bioremediation of aged polycyclic aromatic hydrocarbon-contaminated soil: Link with bioavailability and the dynamics of the bacterial community. Science of the Total Environment, 634, 224-234.

Cevher-Keskin, B., Selçukcan-Erol, Ç., Yüksel, B., Ertekin, Ö., Yıldızhan, Y., Onarıcı, S., \& Memon, A.R. (2018). Comparative transcriptome analysis of Zea mays in response to petroleum hydrocarbon stress. Environmental Science and Pollution Research, 25(32), 32660-32674.

Chaineau, C.H., Morel, J.L., \& Oudot, J. (1997). Phytotoxicity and plant uptake of fuel oil hydrocarbons. Journal of Environmental Quality, 26(6), 1478-1483.

DOF (2002). Norma Oficial Mexicana NOM-021-RECNAT-2000, que establece las especificaciones de fertilidad, salinidad y clasificación de suelos. Estudios, muestreo y análisis. Diario Oficial de la Federación. D.F., México. Recuperado de http://www.profepa. gob.mx/ innovaportal/file/3335/1/ nom-021-semarnat-2000. pdf. Fecha de consulta: 20 de abril del 2015.

DOF (2006). Norma Mexicana NMX-AA-134-SCFI2006, Suelos. Hidrocarburos Fracción Pesada por Extracción y Gravimetría. Método De Prueba. Diario 
Oficial de la Federación. D.F., México. Recuperado de http://legismex. mty.itesm.mx/normas/aa/nmxaa-06/proy-nmxaa-134-scfi-2006.pdf. Fecha de consulta: 17 de mayo del 2016.

García, E., Ferrera, R., Almaráz, J.J., Rodríguez, R., \& García, E. (2013). Biodegradación de Queroseno en la Rizósfera de gramíneas en condiciones de invernadero. Agronomía Costarricense, 37(2), 125-134.

Genty, B., Briantais, J.M., \& Baker, N.R. (1989). The relationship between the quantum yield of photosynthetic electron transport and quenching of chlorophyll fluorescence. Biochimica et Biophysica Acta (BBA)General Subjects, 990(1), 87-92.

Jamil, N., Zairi, M.N.M., Nasim, N.A.I.M., \& Pa'ee, F. (2018). Influences of Environmental Conditions to Phytoconstituents in Clitoria ternatea (Butterfly Pea Flower)-A Review. Journal of Science and Technology, 10(2), 208-228.

Jin, L., Che, X., Zhang, Z., Li, Y., Gao, H., \& Zhao, S. (2017). The mechanisms by which phenanthrene affects the photosynthetic apparatus of Cucumber leaves. Chemosphere, 168, 1498-1505.

Khan, M.A.I., Biswas, B., Smith, E., Naidu, R., \& Megharaj, M. (2018). Toxicity assessment of fresh and weathered petroleum hydrocarbons in contaminated soil-a review. Chemosphere, 212, 755-767.

Khan, S., Afzal, M., Iqbal, S., \& Khan, Q.M. (2013). Plant-bacteria partnerships for the remediation of hydrocarbon contaminated soils. Chemosphere, 90(4), 1317-1332.

Kumar, R., Das, A.J., \& Asha, A.J. (2014). Restoration of Petrol Contaminated Soil by PGPR Consortium Producing Rhamnolipids and Enhancement of Growth and Antioxidant activity of Withania somnifera. Journal of Petroleum and Environmental Biotechnology, S5. 9(8), 44-55. DOI: 10.4172/2157-7463.S5-001

López-Ortiz, C., Ferrera-Cerrato, R., Alarcón, A., Almaraz, J.J., Martínez-Romero, E., \& Mendoza-López, M. (2012). Establecimiento y respuestas fisiológicas de la simbiosis Rhizobium tropici-Leucaena leucocephala en presencia de fenantreno y naftaleno. Revista Internacional de Contaminación Ambiental, 28(4), 333-342.

Lustosa, M.A., López, J.A., Freire, K.C.S., Padilha, F.F., Hernández-Macedo, M.L., \& Cabrera-Padilla, R.Y. (2018). Degradación de hidrocarburos de petróleo por bacterias aisladas de manglares. Revista Peruana de Biología, 25(4), 441-444.

Ma, Y., Rajkumar, M., Rocha, I., Oliveira, R.S., \& Freitas, H. (2015). Serpentine bacteria influence metal translocation and bioconcentration of Brassica juncea and Ricinus communis grown in multi-metal polluted soils. Frontiers in Plant Science, 5, 757.
Maletić, S.P., Dalmacija, B.D., Rončević, S.D., Agbaba, J.R., \& Perović, S.D.U. (2011). Impact of hydrocarbon type, concentration and weathering on its biodegradability in soil. Journal of Environmental Science and Health, Part A, 46(10), 1042-1049.

Díaz-Martínez, M.E.D., Alarcón, A., Ferrera-Cerrato, R., Suarez, J.J.A., \& García-Barradas, O. (2013). Crecimiento de Casuarina equisetifolia (Casuarinaceae) en suelo con diésel, y aplicación de bioestimulación y bioaumentación. Revista de Biología Tropical, 61(3), 1039-1052.

Morales-Guzmán, G., Ferrera-Cerrato, R., Rivera-Cruz, M.C., Torres-Bustillos, L.G., Arteaga-Garibay, R.I., ..., \& Alarcón, A. (2017). Diesel degradation by emulsifying bacteria isolated from soils polluted with weathered petroleum hydrocarbons. Applied Soil Ecology, 121, 127-134.

Muratova, A., Golubev, S., Wittenmayer, L., Dmitrieva, T., Bondarenkova, A., Hirche, F., \& Turkovskaya, O. (2009). Effect of the polycyclic aromatic hydrocarbon phenanthrene on root exudation of Sorghum bicolor (L.) Moench. Environmental and Experimental Botany, 66(3), 514-521.

Onur, G., Yilmaz, F., \& Icgen, B. (2015). Diesel oil degradation potential of a bacterium inhabiting petroleum hydrocarbon contaminated surface waters and characterization of its emulsification ability. Journal of Surfactants and Detergents, 18(4), 707-717.

Pernía, B., Rojas-Tortolero, D., Sena, L., Sisto, A.D., Inojosa, Y., \& Naranjo, L. (2018). Fitotoxicidad de HAP, crudos extra pesados y sus fracciones en Lactuca sativa: una interpretación integral utilizando un índice de toxicidad modificado. Revista Internacional de Contaminación Ambiental, 34(1), 79-91.

Ramadass, K., Megharaj, M., Venkateswarlu, K., \& Naidu, R. (2018). Bioavailability of weathered hydrocarbons in engine oil-contaminated soil: Impact of bioaugmentation mediated by Pseudomonas spp. on bioremediation. Science of The Total Environment, 636, 968-974.

Reddy, P.V., Karegoudar, T.B., \& Nayak, A.S. (2018). Enhanced utilization of fluorene by Paenibacillus sp. PRNK-6: Effect of rhamnolipid biosurfactant and synthetic surfactants. Ecotoxicology and Environmental Safety, 151, 206-211.

Rivera-Cruz, M.C., Trujillo-Narcía, A., Miranda de la, C.M.A., \& Maldonado, C.E. (2005). Evaluación toxicológica de suelos contaminados con petróleo nuevo e intemperizado mediante ensayos con leguminosas. Interciencia, 30(6), 326-331.

Rivera-Cruz, M.D.C., \& Trujillo-Narcía, A. (2004). Estudio de toxicidad vegetal en suelos con petróleos nuevo e intemperizado. Interciencia, 29(7), 369-376. 
Rodríguez-Rodríguez, N., Rivera-Cruz, M.C., TrujilloNarcía, A., Almaráz-Suárez, J.J., \& Salgado-García, S. (2016). Spatial distribution of oil and biostimulation through the rhizosphere of Leersia hexandra in degraded soil. Water, Air, \& Soil Pollution, 227(9), 319.

Sangabriel, W., Ferrera-Cerrato, R., Trejo-Aguilar, D., Mendoza-López, M.R., Cruz-Sánchez, J. S., LópezOrtiz, C., \& Alarcón, A. (2006). Tolerancia y capacidad de fitorremediación de combustóleo en el suelo por seis especies vegetales. Revista Internacional de Contaminación Ambiental, 22(2), 63-73.

SAS Institute Inc. 2002 The SAS system for windows version 9.0 SAS Institute Inc. Cary, North Carolina, USA.

Shiri, M., Rabhi, M., Abdelly, C., \& El Amrani, A. (2015). The halophytic model plant Thellungiella salsuginea exhibited increased tolerance to phenanthreneinduced stress in comparison with the glycophitic one Arabidopsis thaliana: application for phytoremediation. Ecological Engineering, 74, 125-134.

Somtrakoon, K., Chouychai, W., \& Lee, H. (2018). Potential of butterfly pea (Clitoria ternatea) and yam vean (Pachyrhizus erosus) plants for phytoremediation of anthracene- and pyrene-contaminated soil. Songklanakarin Journal of Science and Technology, 40(3), 725-731.

Tan, K.H. \& Nopamombodi, V. (1979). Efect of different levels of Humic acids on nutrient content and growth of corn (Zea mays). Plant and Soil, 51, 283-287.

Totsche, K.U., Danzer, J., \& Kogel-Knabner, I. (1997). Dissolved organic matter-enhanced retention of polycyclic aromatic hydrocarbons soil miscible displacement experiment. Journal of Environmental Quality, 26, 1090-1100.

Trujillo-Narcía, A., Rivera-Cruz, M.D.C., Trujillo-Rivera, E.A., \& Garrigos, A.R. (2018). Reintroducing plant coverage in a tropical wetland contaminated with oil and sulfate: rhizosphere effects on Desulfovibrio populations. Revista de Biología Tropical, 66(2), 908-917.

USEPA-3540C. (1996). Soxhlet extraction organics. SW-846 Test methods for evaluating solid waste physical/chemical methods. Retrieved from http:// www.epa.gov/wastes/azard/testmethods/sw846/ pdfs/3540c.pdf

Volke-Sepúlveda, T., \& Velasco-Trejo, J.A. (2002). Tecnologías de remediación para suelos contaminados. México: INE-SEMARNAT.

Xie, W., Li, R., Li, X., Liu, P., Yang, H., Wu, T., \& Zhang, Y. (2018). Different responses to soil petroleum contamination in monocultured and mixed plant systems. Ecotoxicology and Environmental Safety, 161, 763-768.

Zhang, L., \& Xing, D. (2008). Rapid determination of the damage to photosynthesis caused by salt and osmotic stresses using delayed fluorescence of chloroplasts. Photochemical \& Photobiological Sciences, 7(3), 352-360.

Zhu, L., Wang, Y., Jiang, L., Lai, L., Ding, J., Liu, N., \& Rimmington, G.M. (2015). Effects of residual hydrocarbons on the reed community after 10 years of oil extraction and the effectiveness of different biological indicators for the long-term risk assessments. Ecological Indicators, 48, 235-243. 